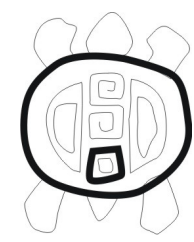

RMA

Arqueología

\title{
Agricultura y potencial productivo en el Valle de Ambato, Catamarca, Argentina (siglos VI a XI d.C)
}

\author{
Germán G. Figueroa \\ Museo de Antropología, Facultad de Filosofía y Humanidades, \\ Universidad Nacional de Córdoba - SECYT-UNC. E-mail: gfigueroa@argentina.com
}

\begin{abstract}
Resumen
El control y acumulación de recursos económicos en el Valle de Ambato entre los siglos VI y XI d.C. ha sido considerado como un factor de suma importancia en el mantenimiento y reproducción de las diferencias sociales entre las personas. Se cree que el uso de sectores seleccionados del paisaje como áreas de producción agrícola se hallaba vinculado de manera funcional y organizacional con agregados residenciales o aldeas emplazadas en el fondo del valle. En dichos sectores, más precisamente sobre los faldeos de la sierra de la Graciana y del Ambato, se registró la presencia de infraestructura agrícola, tales como terrazas de cultivo, represas y canales. En este trabajo, se analizan los sistemas de cultivo y su potencial productivo en relación a los grupos humanos que habitaron el valle, para lo cual se lleva a cabo una caracterización del sistema hidro-agrícola y un análisis de su capacidad sustentadora en base a registros arqueológicos y observaciones etnográficas propias. Los resultados obtenidos permitirán discutir acerca de la posible existencia de un excedente económico, utilizado por un determinado sector de la población con el propósito de usufructuar los beneficios derivados del mismo.
\end{abstract}

Palabras clave: Valle de Ambato, Aguada, sistemas agrícolas, potencial productivo, excedente económico.

Agriculture and productive potential in the Ambato Valley, Catamarca, Argentina (VI-XI centuries AD).

\begin{abstract}
The control and accumulation of economic resources in the Ambato Valley between VI and XI centuries AD has been considered a key factor in the maintenance and reproduction of social differences between people. It is believed that the use of selected sectors of the landscape as agricultural production areas was functional and organizational associated with villages located in the valley bottom. In these sectors, and more precisely on the slopes of Graciana and Ambato mountain ranges, the presence of agricultural infrastructure was registered, such as farming terraces, dams and channels. In this paper, we analyze the farming systems and their productive potential in relation to the human groups that inhabited the valley. In order to accomplish these objectives we carry out a characterization of the hydro-agricultural system and an analysis of its carrying capacity based on archaeological records and our own ethnographic observations. The obtained results will allow discussing about possible existence of an economic surplus, used by a particular sector of the population with the purpose of usufruct benefits derived from it.
\end{abstract}

Keywords: Ambato Valle, Aguada, agricultural systems, productive potential, economic surplus.

Alrededor del siglo VI d.C., se registra en el Valle de Ambato, Catamarca, Argentina, una serie de innovaciones y algunas combinaciones de antiguos elementos en nuevas configuraciones, contrastantes con las formas organizativas y modos de hacer las cosas en el pasado anterior, que se integran entre sí como componentes de una nueva organización más compleja que la anterior (Laguens 2004, 2006). Este nuevo modo de vida estuvo fundado en una intensificación de la economía, asociada a un incremento marcado de la población, con diversificación de roles sociales y una incipiente especialización artesanal, junto con desigualdades sociales y políticas (Assandri 2006; Laguens 2004; Marconetto 2008, 2006; Pérez Gollán 1991, 2000). En este marco, la organización y el manejo de la producción de recursos para la subsistencia, incluyendo las tierras y el agua, pudo haber sido un factor clave para la acumulación y el mantenimiento del poder y la acentuación de las diferencias entre las personas. Por lo tanto, entre otras alternativas, es posible pensar que la inversión y utilización de sectores seleccionados del paisaje como áreas de producción agrícola, vinculados de manera organizacional y funcional con agregados residenciales o aldeas emplazadas en el fondo de valle (Assandri y Laguens 2003), desempeñaron un rol esencial en la generación de un excedente económico.

En esta investigación se pretende indagar, puntualmente, sobre el potencial productivo y la capacidad de carga 
humana de los sistemas agrícolas en el valle durante la época Aguada. En consecuencia, este trabajo se estructurará en dos secciones claramente definidas. En la primera de ellas se llevará a cabo una caracterización de la infraestructura agrícola, localizada sobre las estribaciones de la sierra del Ambato, para lo cual se tendrá en cuenta la información emanada de las prospecciones efectuadas durante los años 2006 y 2007. En la restante se realizará una primera aproximación a la estimación de la escala de producción en términos de rendimiento y capacidad sustentadora de los sistemas agrícolas, mediante la utilización de datos reunidos en entrevistas etnográficas, efectuadas a campesinos residentes en la zona bajo estudio, y el empleo de cálculos matemáticos. Sobre este último punto, es necesario mencionar que no se intenta agotar la problemática a investigar ni la complejidad de los factores que articuladamente dirigen la dinámica histórica de las sociedades, lo que sí se pretende es elaborar un marco referencial adecuado que permita aportar nuevos elementos para el enriquecimiento de las discusiones e hipótesis sobre este tema.

\section{Algunas consideraciones teórico-metodológicas}

A lo largo del tiempo la etnoarqueología' fue objeto de fuertes y variadas críticas, sin embargo en la actualidad numerosos investigadores coinciden en afirmar que su potencial en el terreno arqueológico es enorme (Binford 1978; Borrero y Yacobaccio 1989; Hernando Gonzalo 1995; Kuznar 2001; Nielsen 1997; Yacobaccio et al. 1998; entre otros). Según Politis (2004), la crítica más común efectuada a la etnoarqueología ronda en torno a la dificultad de extrapolar la información actualística hacia sociedades del pasado, lo cual se debe, fundamentalmente, a que aún no están adecuadamente desarrolladas las bases epistemológicas para llevar a cabo esta transmisión. Durante años este tema generó profundos debates, pero actualmente a partir del análisis realizado por Wylie (1982, 1985), existe entre los arqueólogos la aceptación de la argumentación analógica como un proceso válido de interpretación del registro arqueológico. Por otro lado, algunos investigadores han planteado que las sociedades del presente no pueden servir de referentes análogo de las sociedades pasadas. Esta crítica actualmente es considerada injustificada por Politis $(2002,2004)$, ya que la investigación etnoarqueológica opera bajo los principios de la argumentación analógica y por lo tanto, los dos elementos de la analogía (fuente y sujeto) no deben ser iguales (en cuyo caso no sería necesario un razonamiento analógico) sino que deben tener ciertas condiciones de comparabilidad. En definitiva, la fortaleza de la analogía generada a partir de la etnoarqueología no reside en el grado de semejanza entre la fuente (la sociedad presente)

\footnotetext{
1 Para O'Connell (1995), la etnoarqueología puede ser definida como el campo del conocimiento que estudia las relaciones entre el comportamiento humano y sus consecuencias materiales en el presente, con la intención de identificar patrones que expliquen esas vinculaciones, pudiendo, así, aplicar estos conocimientos a la interpretación de la conducta humana pasada.
}

y el sujeto (la sociedad pasada percibida a través del registro arqueológico) sino en la estructura lógica de la argumentación y en la similitud entre los términos de la relación. Lógicamente, cuanto mayor es el grado de semejanza entre la fuente y el sujeto, la argumentación analógica tiene un mayor potencial, pero este nivel de semejanza por sí mismo no garantiza de modo alguno la solidez de la argumentación ni la autenticidad de los enunciados (Politis 2002, 2004).

Asimismo, debe destacarse que más allá de que se consideren válidas las diversas formas en que se concibe la etnoarqueología (Binford 1983; Hernando Gonzalo 1995; Hodder 1982; etc.), en este trabajo se atiende, principalmente, a lo propuesto por O'Connell (1995), quien plantea que el foco de esta disciplina puede ser ampliado incluyendo el estudio de la variabilidad del comportamiento humano, bajo el marco de la Ecología evolutiva, ya que esta teoría cuenta con el potencial de explicar esta variabilidad bajo circunstancias más vastas. Por lo tanto, el punto sustancial consistiría en la explicación de pautas de comportamiento a través de la identificación de las restricciones que lo limitan, especialmente, aquellas que afectan el éxito reproductivo, y una predicción de un patrón óptimo del comportamiento, que posteriormente será corroborado con el registro arqueológico. Sintetizando, la firmeza de esta teoría radica en la capacidad de generar predicciones sobre la variabilidad en un vasto espectro de fenómenos y proveer de una estructura adecuada en donde integrarlos. De este modo, es posible obtener expectativas potencialmente comprobables sobre el comportamiento y sus consecuencias arqueológicas en cualquier asentamiento, pasado o presente, aunque sea distinto a los conocidos entre los humanos modernos (O'Connell 1995).

Las técnicas empleadas en la recolección de información consistieron en entrevistas estructuradas llevadas a cabo con campesinos residentes en la zona bajo estudio, observaciones participativas y entrevistas abiertas. En tanto, el modelo empleado para estimar la capacidad de carga de la agricultura requirió: (1) identificar los principales recursos alimenticios de los que dispusieron los antiguos habitantes de estos espacios y estimar su productividad; (2) calcular el área bajo cultivo durante la época Aguada; y (3) estimar la naturaleza de la dieta prehispánica en términos de requerimientos de calorías. Este modelo, ideado por Treacy (1994), arroja como resultado una capacidad máxima de carga y no pretende de modo alguno lograr una cifra que contenga el mismo grado de precisión que la obtenida por ejemplo por historiadores demógrafos para épocas históricas. En decir, el concepto de capacidad de carga es un razonamiento heurístico, en su sentido más estricto, y la principal utilidad de la estimación de la producción se centra en demostrar las relaciones generales existentes entre terreno, población y la naturaleza del cambio agrícola (Treacy 1994). Para finalizar, resulta oportuno mencionar que el uso de cál- 
culos matemáticos para estimar el potencial agrícola en sociedades prehispánicas, constituye una línea de trabajo que se inició hace casi cuatro décadas atrás y que aún hoy goza de gran aceptación entre la comunidad arqueológica de diferentes partes del mundo (Cruz y Palerm 2001; Dickson 1980; Erickson 1992; Laguens 1999; Moseley et al. 2005; Raffino 1973; Treacy 1994; entre otros).

\section{Área de estudio}

El Valle de Ambato se ubica a $70 \mathrm{~km}$ de la ciudad de Catamarca, dentro del Departamento que lleva el mismo nombre. Sus límites están establecidos por las Sierras Graciana-Balcozna al Este y la del Ambato-Manchao al Oeste. En su porción Norte corre serpenteante el río de Los Puestos o Nacimientos, que baja de los altos de Singuil, cambiando luego de nombre, por el de río del Valle -Figura 1- (de la Orden y Quiroga 1997). Toda esta zona se encuentra influenciada por un clima continental cálido, con precipitaciones anuales que oscilan entre los 350 y 800 mm, las cuales se concentran en la estación estival, más específicamente entre los meses de enero y febrero (Saravia Toledo 1995).
En la cuenca del río de Los Puestos, área considerada en este trabajo, la vegetación se dispone en "cinturones o pisos", cada uno de los cuales exhibe una estructura y composición determinada. Los rangos altitudinales ocupados por cada piso varían en función de la latitud, de la longitud y de los contextos microclimáticos, como por ejemplo la orientación de las laderas. De este modo, pueden distinguirse tres pisos (Marconetto 2006; Morlans y Guichon 1995):

a) Bosque serrano: se sitúa entre los 800 y los 1500 msnm. Algunas de las especies propias de este piso son algarrobo blanco (Prosopis alba), algarrobo negro (Prosopis nigra), coco (Fagara coco), orco quebracho (Schinopsis haenkeana) y viscote (Acacia visco). En las laderas con exposición hacia el Este y hacia el Sur, con un microclima mucho más húmedo, el bosque es de mayor densidad y asciende un poco más que aquellos con exposición hacia el Norte.

b) Arbustal-pastizal: se encuentra entre los 1500 hasta los 1800 msnm. En este piso, predominan distintas especies de los géneros Stipa, Festuca Borthriocloa y Piptochaetium, y especies arbustivas tales como palo amarillo (Aloysia gratisima) y barba de tigre (Collettia spinosissima).

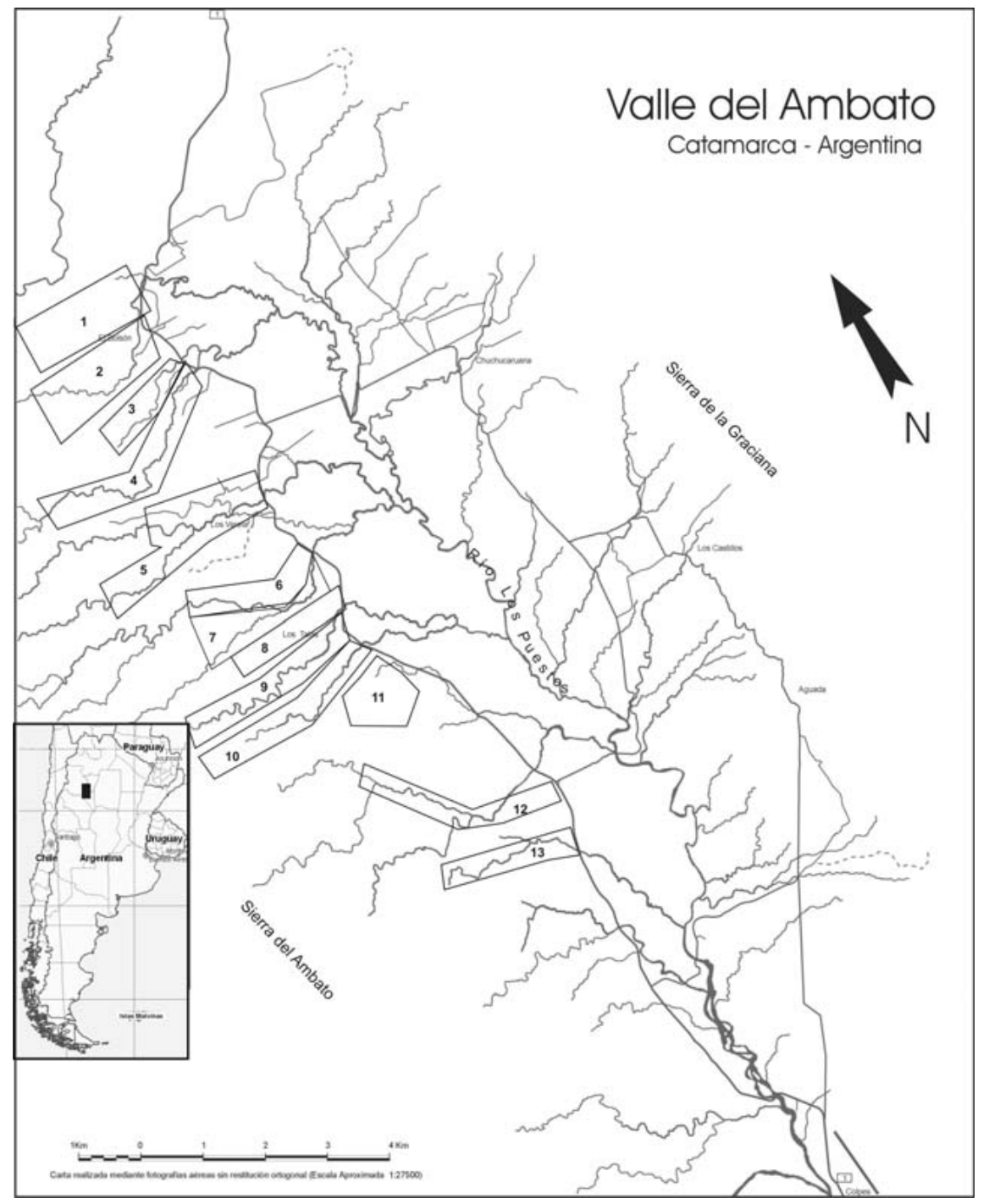

Figura 1: Plano del Valle de Ambato. Las áreas enumeradas corresponden a las zonas donde fue posible identificar estructuras vinculadas a la producción agrícola: 1- Los Bustamente, 2- El Bolsón, 3- Los Reinoso 1, 4- Los Reinoso 2, 5- Los Varela, 6- Los Potrerillos A, 7- Los Potrerillos B, 8Cárdenez, 9- La Quebrada, 10- La Gruta, 11-Carrazán, 12-Saavedra y 13-Luchi. 
c) Pastizal de altura: se ubica por sobre los 1800 a 2000 msnm, aquí prácticamente desaparecen las leñosas y la vegetación se limita a una asociación de gramíneas entre las que se encuentran Stipa ichu, Stipa tenuísima y Festuca hieronymii.

\section{Condiciones paleoambientales en el área de estudio}

A lo largo del tiempo, en los Andes centrales y meridionales, las investigaciones arqueológicas han reunido información sobre los climas del pasado y su incidencia en la conformación del paisaje natural y cultural, gracias al aporte de diversos estudios paleoambientales (Abbot et al. 1997; Baied 1999; Binford et al. 1997; Markgraff 1985; Ratto 2003; Thompson et al. 1994; Valero Garcés et al. 2000; entre otros), derivados del análisis de secuencias polínicas, indicadores paelolacustres y núcleos de hielo (Marconetto 2008) (Tabla 1).

Los primeros trabajos que proporcionaron información paleoambiental sobre el NOA, fueron los análisis realizados por Markgraf (1985), a partir de polen y carbón provenientes del perfil de una turba del sitio El Aguilar, Puna de Jujuy. Estos estudios permitieron determinar que para momentos anteriores al $12.000 \mathrm{AP}$, imperó un clima más frío y seco que en la actualidad. En tanto, estableció que para el Holoceno, se sucedieron al menos tres intervalos paleoambientales. En el primero, desarrollado entre 10.000 y $7.500 \mathrm{AP}$, imperaron condiciones frías y húmedas, junto con el establecimiento del régimen de Iluvias estivales. En el segundo, ocurrido entre 7.500 y 4.000 AP, el clima varió a condiciones más cálidas y áridas. Finalmente, en el tercero, acontecido entre el 4.000 AP y $500 \mathrm{AP}$, se establecieron las condiciones ambientales semejantes a las actuales.

Similares resultados fueron obtenidos por Baied (1999), en base al estudio de diagramas de polen de comunidades del género Polylepis, en distintos lugares de los Andes de Argentina, Bolivia, Chile y Perú. Asimismo, Baied (1999) y Markgraf (1985) coinciden en que a partir del 4000 AP comenzó a establecerse la zonación vegetal actual.

Por otro lado, en Chaschuil, Puna de Catamarca, se

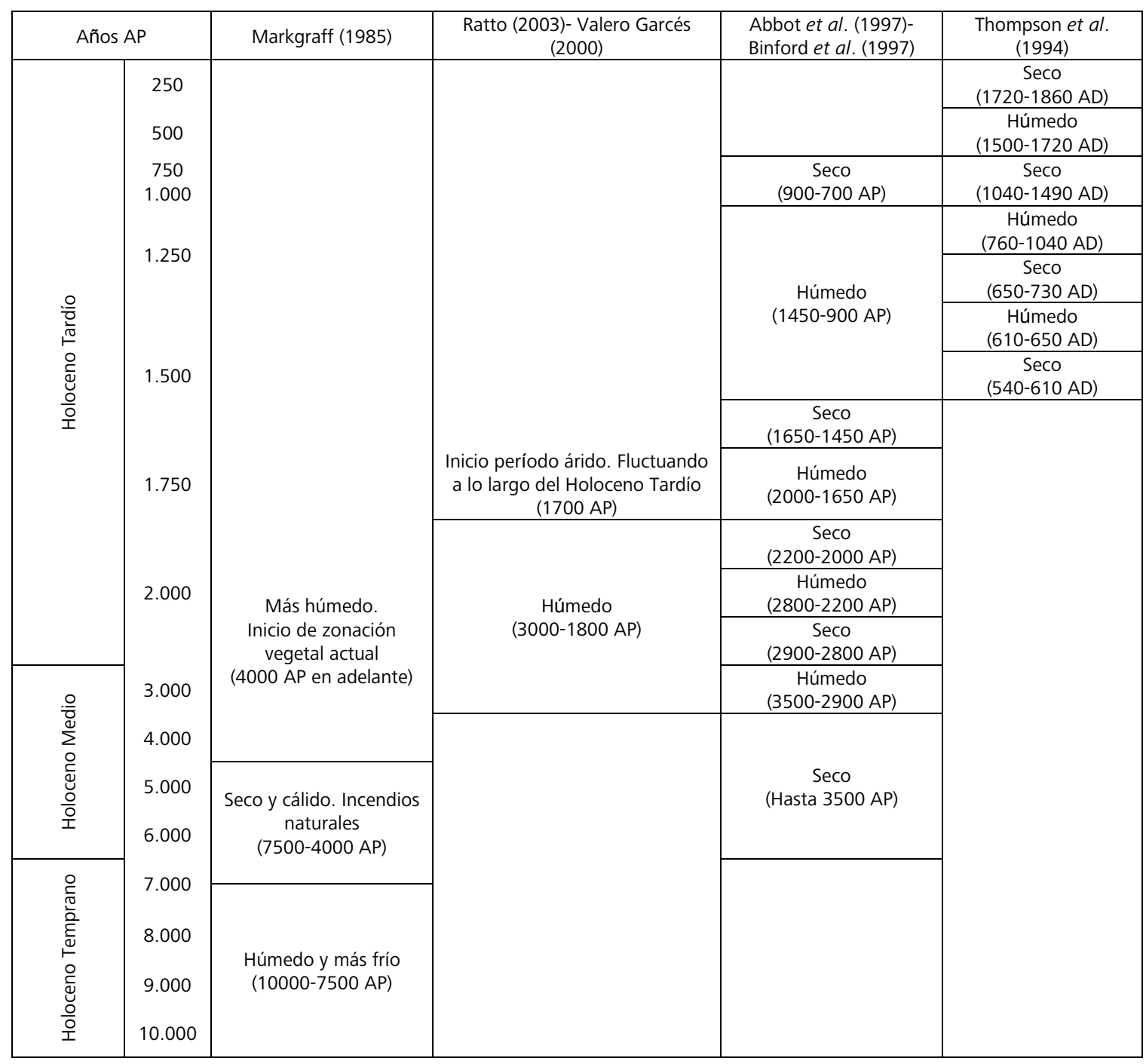

Tabla 1: Síntesis de los estudios paleoambientales en la zona andina (Adaptado de Marconetto 2008). 
realizaron estudios de diatomeas, polen y sedimentos lacustres, que permitieron modelar la evolución ambiental de la región y aportar datos sobre las fluctuaciones de las condiciones de humedad a lo largo del Holoceno Tardío. Los registros obtenidos permitieron plantear que la región puneña de Chaschuil podría haber contado con un período húmedo entre el 3.000 y 1.800 AP, seguido por un período árido. En tanto, se pudo establecer que los períodos secos fluctuaron a lo largo del Holoceno Tardío, mientras que la máxima expresión húmeda se sucedió entre los siglos XVII y XIX (Ratto 2003). Esta información resultó compatible con otros registros lacustres de latitudes similares, de la vertiente occidental de los Andes en Chile (Valero Garcés et al. 2000).

Según Marconetto (2008) estas oscilaciones de períodos de humedad y aridez se registraron en toda el área andina, lo cual puede observarse a través de los registros tomados en distintos puntos de los Andes. Por lo tanto, esta investigadora considera que estas fluctuaciones debieron también sucederse también en el Valle de Ambato, más allá de posibles variaciones locales. Además, las identificaciones realizadas sobre material vegetal de fogones domésticos de sitios arqueológicos, revelaron que los taxones identificados corresponden a la misma formación vegetal que se encuentra actualmente en el Valle de Ambato (Marconetto 2008).

\section{Estado de las investigaciones}

La primera referencia sobre centros agrícolas Aguada en la provincia de Catamarca se remonta al trabajo de Ardissone (1944), quien examinó los sistemas de andenerías localizados en Las Juntas y Trancas, en la región media de Catamarca. Varias décadas después, Kriscautzky (1995, 2000a, 2000b) analizó y efectuó una clasificación de estructuras agrícolas pertenecientes a ocupaciones Aguada, ubicadas, todas ellas, en la cuenca del río Las Juntas y Quebrada del Tala. De igual manera, en el Valle de Catamarca, Puentes $(2003,2005)$ llevó a cabo tareas de prospección y relevamiento en un sistema agrícola

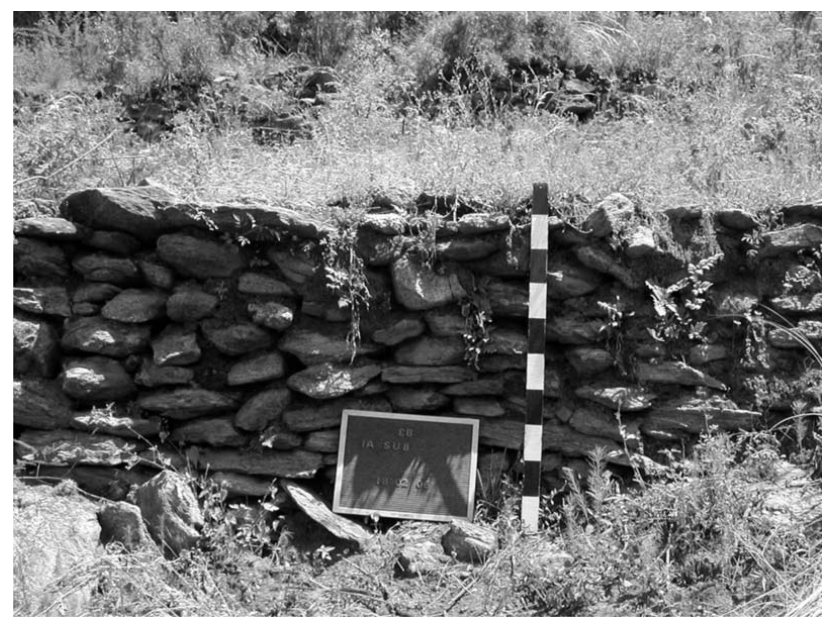

Figura 2: Terraza de ladera recta. emplazado en el sector central de la Sierra de Ambato, más precisamente en la ya mencionada Quebrada del Tala.

En lo que respecta específicamente al Valle de Ambato, escasos han sido los trabajos de investigación que centraron su atención en la infraestructura agrícola y el manejo del agua. Herrero y Ávila (1993), a partir de prospecciones realizadas en el piedemonte de la Sierra de la Graciana, describieron un conjunto de sistemas agrícolas, a los que caracterizaron de baja inversión y los vincularon a sitios correspondientes al periodo Formativo. Posteriormente, nuevos trabajos de campo consumados en el sector sur y centro de la Sierra de Ambato permitieron identificar la presencia de un considerable conjunto de estructuras agrícolas, así como también de un reducido número de canales de riego (Cruz 2004; Hierling 2000).

En cuanto al material arqueobotánico identificado para el periodo en estudio, es necesario señalar que a través de excavaciones estratigráficas efectuadas en los sitios El Altillo y Piedras Blancas, ambos emplazados en el fondo del valle, se han podido registrar restos carbonizados de diversas especies cultivadas (Laguens 2004). Se recuperaron granos aislados y marlos de maíz subglobosos -Zea mays-, de 5 a 7 mm de diámetro, que por su forma y tamaño corresponderían tanto a la raza Microsperma -Maíz Reventón- denominado vulgarmente como Maíz Perla o Rosita, como a la raza Oryzaea -Maíz Pisingallo-. Además, se han identificado restos de poroto -Phaseolus vulgaris- (Pochettino 2000).

\section{Caracterización de los sistemas agrícolas de la sierra del Ambato}

Los sistemas agrícolas de la vertiente occidental del Valle de Ambato, se integran en sistemas independientes a partir de un cauce colector como eje sobre el cual se fueron aterrazando o nivelando los tributarios secundarios y las laderas laterales. De este modo, puede observarse, entre los 1.122 y 1.580 msnm, un extenso espacio destinado a la producción agrícola, el cual se halla compuesto por terrazas de cultivo, recintos, represas y canales.

En base a las propuestas de Albeck (1993), Denevan (1980), Donkin (1979), Raffino (1973) y Treacy (1994), quienes toman en cuenta atributos tales como emplazamiento, grado de pendiente, morfología, técnicas de construcción y materia prima, fue posible registrar dos grandes clases de terrazas de cultivo (Figueroa 2008a):

1) Terrazas de ladera: Se encuentran emplazadas sobre los flancos interfluviales, siendo las más comunes en la cuenca del río de Los Puestos. A partir de las características del muro de contención y la pendiente, se las pudo discriminar en dos subclases: rectas (Figura 2) y de contorno (Figura 3). La primera de ellas, corresponde a terrazas que presentan muros básicamente lineales, los cuales cortan 


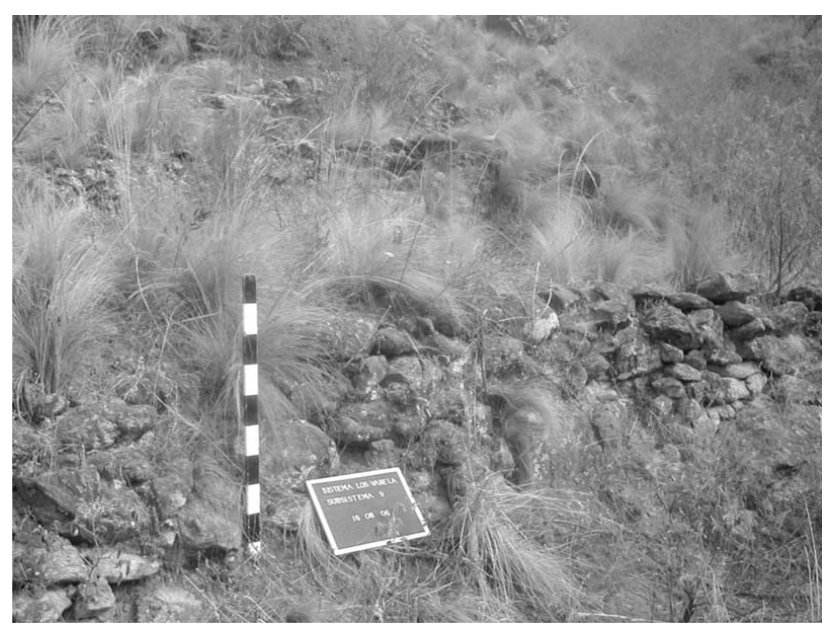

Figura 3: Terraza de ladera de contorno.

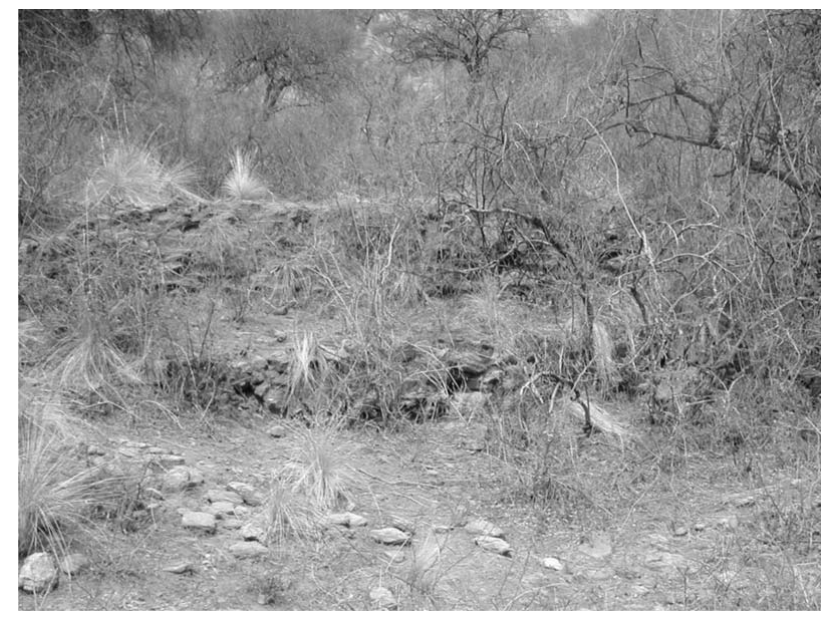

Figura 4: Terraza de cauce.

transversalmente las pequeñas quebradas de los cerros. Las restantes, poseen como rasgo característico que sus muros continúan o se aproximan a las oscilaciones del terreno, localizándose siempre en los relieves positivos.

2) Terrazas de cauce: Son menos numerosas que las anteriores y sus muros dispuestos en forma de escalera, aproximadamente a $10 \mathrm{~m}$ unos de otros, se sitúan transversalmente en los arroyos temporarios del río de Los Puestos (Figura 4).

De la totalidad de las estructuras agrícolas identificadas (2398), el 63,47\% corresponde a terrazas de laderas rectas, el 31,20\% a terrazas de ladera de contorno y el $5,33 \%$ a terrazas de cauce.

Por otra parte, entre las terrazas de cultivo se destaca, ya sea por su abundancia como por su disposición espacial, la presencia de 71 recintos. Estas estructuras pueden hallarse adosadas directamente a los aterrazamientos o bien en sus inmediaciones, en una estrecha proximidad. Considerando su ubicación, morfología, técnica de manufactura y materiales de construcción, se pudieron discriminar en tres clases de construcciones:
1) Recintos de planta circular con muro de piedra simple: Estas pequeñas construcciones, que en promedio no exceden los 2,61 m de diámetro, se ubican en la cima o en la ladera de los cerros, encontrándose, generalmente, insertas entre las terrazas de contorno o bien entre otras clases de estructuras. Se identificaron siete recintos correspondientes a esta clase (Tabla 2).

2) Recintos de planta rectangular con muro de piedra simple: Estas estructuras presentan un módulo principal de forma rectangular, el cual se halla subdividido internamente en uno o dos recintos. Aquí, la técnica constructiva empleada corresponde a la denominada pared de tapia con columnas de piedras (Assandri 2006). Dichas construcciones, usualmente, se sitúan en los sectores planos del piedemonte y de las laderas, en proximidad de las terrazas de ladera rectas y de contorno, aunque nunca inmediatamente junto a ellas. Los trabajos de campo permitieron reconocer 19 de ellas (Tabla 2).

3) Recintos de planta rectangular o circular con muro de piedra doble: Esta clase de estructuras exhibe como característica principal una doble hilera de piedras rectangulares, las cuales fueron fijadas en la tierra verticalmente. En lo que se refiere a su ubicación, se logró establecer que no siguen un patrón fijo, ya que pueden hallarse de manera aislada en la cumbre de los cerros o asociadas a otras clases de recintos en la ladera de los mismos. En este último caso, pudo observarse que en ocasiones comparten su muro a modo de medianera con las terrazas de contorno. En total se registraron 35 de estas unidades (Tabla 2).

Para los primeros, mediante trabajos de excavación se determinó que estuvieron destinados al almacenamiento de productos agrícolas (Cruz 2004; Figueroa 2008b). Mientras que, para los recintos de planta rectangular de muro simple, se hipotetizó que pudieron haber funcionado como unidades habitación que albergaron a individuos vinculados a las labores agrícolas (Figueroa 2008b). Por último, para la tercera clase de estructuras se estableció que funcionaron como corrales (Dantas y Figueroa 2009).

La infraestructura hidráulica descubierta hasta la actualidad comprende tres tramos de canales y cuatro represas. Los canales fueron construidos a través de la utilización de piedras lajas clavadas de canto en la tierra y se sitúan en proximidad a las terrazas de contorno y recintos. Mediante su relevamiento se logró constatar que bajo ninguna circunstancia sus trazados se asocian a las superficies aterrazadas, por lo cual es posible plantear que la función de estas estructuras en el pasado no fue la de irrigar estos terrenos de cultivo. Además, si se atiende a su ubicación y orientación se puede hipotetizar que probablemente habrían sido construidos con la intención de transportar agua desde las nacientes de los afluentes del río de Los Puestos hacia el fondo del valle, donde se encuentran ubicados los poblados. En cambio, todas las 


\begin{tabular}{lccccc}
\hline Unidad de prospección & \multicolumn{4}{c}{ Clases de recintos } & Total \\
\cline { 2 - 5 } & $\begin{array}{c}\text { Circulares con muro } \\
\text { de piedra simple }\end{array}$ & $\begin{array}{c}\text { Rectangulares con } \\
\text { muro de piedra } \\
\text { simple }\end{array}$ & $\begin{array}{c}\text { Circulares o } \\
\text { rectangulares con } \\
\text { muro de piedra doble }\end{array}$ & No clasificados & Recintos \\
\hline Los Bustamante & 1 & - & - & 1 & 2 \\
Los Reinoso 2 & 1 & 3 & 1 & - & 5 \\
Los Varela & 1 & 8 & - & - & 20 \\
Los Potrerillos A & - & - & 4 & 3 & 9 \\
Los Potrerillos B & - & - & 13 & 2 & 6 \\
Cárdenez & 2 & - & - & 1 & 16 \\
Carrazán & 2 & 2 & - & - & 8 \\
Saavedra & - & 2 & - & 1 & 2 \\
El Bolsón & - & 19 & 35 & 10 & 71 \\
\hline Totales & 7 & & & & 3 \\
\hline
\end{tabular}

Tabla 2: Clases y cantidad de recintos identificados en la ladera del Ambato.

represas registradas se ubican en el lecho de los arroyos, donde aflora la roca base. Una particularidad que presentan estas estructuras es que fueron edificadas con muro de piedra doble relleno de tierra, distinguiéndose así de las terrazas de cauce, las que sin excepción exhiben muro simple. De este modo, se puede plantear que la agricultura practicada habría dependido exclusivamente de los aportes pluviales estacionales y del manejo de aguas de escorrentías. La construcción de represas, terrazas de ladera rectas y de cauce, todas ellas ubicadas sobre relieves negativos del terreno, estarían indicando la utilización de una tecnología vinculada, específicamente, al manejo de agua de escorrentías (Figueroa 2008a).

En el interior y en los alrededores de las terrazas de cultivo, recintos y estructuras hidráulicas se recuperaron un total de 249 artefactos, de los cuales 84 corresponden a materiales líticos y 165 a cerámicos.

Mediante el análisis y clasificación de la alfarería, fue posible efectuar comparaciones con otros conjuntos cerámicos de Ambato, como por ejemplo los provenientes de los sitios El Altillo y Piedras Blancas, asociados al periodo Formativo y Aguada, respectivamente (Fabra 2002). El propósito de este estudio residió, principalmente, en tratar de identificar pautas o patrones que permitieran realizar hipótesis que contengan implicancias cronológicas. Sobre este tema, resulta conveniente destacar que una recolección sistemática de la cerámica de superficie, puede proporcionar información importante acerca de la utilización de un mismo terreno de cultivo en lapsos temporales disímiles, además de resultar diagnóstica del grupo social que construyó las estructuras agrícolas o hizo uso de ellas (Albeck 2003/2005). Los resultados obtenidos permitieron observar, más allá de lo escasa y fragmentada de la muestra, una gran diversidad en cuanto a técnicas especiales de decoración, acabado de superficie, grosor de pared, dureza, antiplástico y tamaño de las cavidades. Siguiendo estas variables, ya contempladas con anterioridad por Fabra (2002), se logró establecer que el 18,18\% de los fragmentos corresponden a clases cerámicas preAguada y el $81,82 \%$ a clases Aguada. Dentro de esta última, fue posible reconocer siete grandes variedades: negro pulido, rojo alisado/pulido, naranja alisado, castaño/rojo alisado, castaño pulido, crema alisado y toscos (Fabra 2006) -Tabla 3-. Entonces, si se considera a estos artefactos como posibles indicadores culturales y cronológicos (Albeck 2003/2005; Erickson 1980; Treacy 1994), se puede plantear que estas estructuras pudieron haber funcionado simultáneamente, durante algún momento de la etapa Aguada.

El material lítico hallado comprende tanto desechos de talla $(\mathrm{N}=50)$ como instrumentos $(\mathrm{N}=34)$. En cuanto a los primeros, se pudo constatar que estarían vinculados a etapas iniciales de talla, es decir, a la reducción de núcleos mediante percusión directa en cuarzo. En relación a los segundos, se logró observar que la mayoría estarían ligados

\begin{tabular}{|c|c|c|c|c|c|c|c|c|}
\hline & Negro Pulido & Rojo Alisado/Pulido & $\begin{array}{l}\text { Naranja } \\
\text { Alisado }\end{array}$ & $\begin{array}{c}\text { Castaño/Rojo } \\
\text { Alisado }\end{array}$ & $\begin{array}{l}\text { Castaño } \\
\text { Pulido }\end{array}$ & $\begin{array}{l}\text { Crema } \\
\text { Alisado }\end{array}$ & Tosco & Total \\
\hline El Bolsón & - & - & - & - & - & - & $100,00 \%$ & $100 \%$ \\
\hline Los Reinoso & $60,00 \%$ & - & - & - & - & - & $40,00 \%$ & $100 \%$ \\
\hline Los Varela & $54,54 \%$ & $4,55 \%$ & - & $2,27 \%$ & - & - & $38,64 \%$ & $100 \%$ \\
\hline Los Potrerillos & $21,05 \%$ & - & - & - & - & $5,27 \%$ & $73,68 \%$ & $100 \%$ \\
\hline Cárdenez & - & - & $25,00 \%$ & - & - & - & $75,00 \%$ & $100 \%$ \\
\hline Carrazán & - & - & $8,33 \%$ & - & $8,33 \%$ & - & $83,33 \%$ & $100 \%$ \\
\hline Saavedra & - & - & $15,00 \%$ & - & $5,00 \%$ & - & $80,00 \%$ & $100 \%$ \\
\hline
\end{tabular}

Tabla 3: Distribución porcentual de cerámica Aguada en los sitios arqueológicos ubicados en la ladera del Ambato. 


\begin{tabular}{|c|c|c|c|c|c|c|c|c|c|c|c|}
\hline & 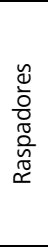 & $\frac{\frac{\tilde{u}}{0}}{\frac{\tilde{d}}{z}}$ & 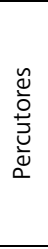 & 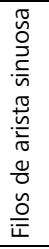 & 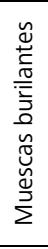 & 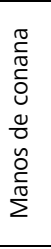 & 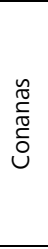 & 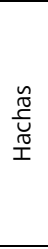 & 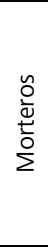 & 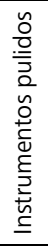 & $\begin{array}{l}\frac{\tilde{d}}{\pi} \\
\stackrel{0}{\circ}\end{array}$ \\
\hline El Bolsón & 1 & 2 & 1 & 1 & - & 1 & - & - & - & 1 & 7 \\
\hline Saavedra & - & - & 1 & - & 1 & - & - & 1 & - & - & 3 \\
\hline Carrazán & - & 1 & 1 & - & - & 3 & 1 & - & 2 & - & 8 \\
\hline Cárdenez & - & - & 1 & - & - & - & - & - & - & - & 1 \\
\hline Los Reinoso & - & - & - & - & - & - & - & - & 5 & - & 5 \\
\hline Los Varela & - & - & - & - & - & - & 2 & - & 8 & - & 10 \\
\hline Total & 1 & 3 & 4 & 1 & 1 & 4 & 3 & 1 & 15 & 1 & 34 \\
\hline
\end{tabular}

Tabla 4: Cantidad y clases de artefactos líticos registrados por sitios.

a actividades inherentes al procesamiento de productos vegetales, como por ejemplo, conanas, manos de moler y morteros (Tabla 4). En esta ocasión la materia prima involucrada corresponde a gneis (71\%) y cuarzo $(29 \%)$.

En síntesis, en la zona bajo estudio, las diferentes estructuras identificadas se combinan entre sí conformando un patrón recurrente en lo que respecta a construcción y ubicación espacial. En lo referido a la técnica constructiva, se logró determinar que la mayoría de las estructuras fueron edificadas con piedra seca y sólo unas pocas mediante la utilización de una argamasa o ligante de barro. En todos los casos, la materia prima seleccionada se restringió a rocas metamórficas tales como esquisto, gneis, migmatita y clastos de cuarzo, las cuales se hallan disponibles en toda el área, a corta distancia de los aterrazamientos. En tanto, la disposición espacial de las estructuras posee la cualidad de ser sumamente uniforme, llamando la atención la escasa diversidad en lo que respecta a lugares de emplazamiento y modos de articulación entre recintos y terrazas (Tabla 2). Sobre este último punto, se logró observar la presencia de terrazas de contorno en casi todas las laderas, las cuales se ven interrumpidas en su ordenamiento por recintos que se ubican entre y sobre ellas. En las quebradas se encuentran las terrazas de muro recto y por debajo de ellas, donde la topografía lo permite, las de cauce.

\section{Enfoque etnográfico de la investigación}

Para calcular la capacidad de carga de los sistemas agrícolas, resultó indispensable la obtención de un cuerpo de datos, debidamente sustentado, acerca del potencial productivo y de las diferentes estrategias que involucran las prácticas agrícolas en el área de estudio. Inicialmente, se planteó la posibilidad de utilizar como marco referencial para la estimación de la productividad de los recursos cultivables reconocidos arqueológicamente en Ambato, datos provenientes de otras zonas geográficas (Barlow 2002; Dickson 1980; Laguens 1999; Muscio 2004; Raffino 1973; Treacy 1994). Sin embargo, ante la presencia de diversos factores climáticos y culturales, que son propios de cada región y que no se pueden ser fácilmente trasla- dables a otras (i.e. temperatura, evaporación, radiación solar, precipitaciones, humedad, vientos, tipo de suelo y modalidades de cultivo), se decidió tratar de minimizar el margen de error mediante la realización de observaciones etnográficas propias.

Los trabajos de campo propiamente dichos se llevaron a cabo desde comienzos de 2006 hasta finales de 2007, coincidiendo en ocasiones con la época de siembra y cosecha del poroto y del maíz. Se entrevistaron 12 agricultores (Tabla 5), oriundos de las localidades rurales de Los Varela, Los Talas, Los Castillos y El Bolsón, todas ellas situadas en el departamento Ambato, provincia de Catamarca. Las entrevistas fueron realizadas a informantes calificados del lugar, los cuales practican una agricultura a escala doméstica y desprovista de tecnología moderna. Se entiende por calificados a aquellos individuos que se destacan en su comunidad por su conocimiento, así como quienes a lo largo de los años en diálogos informales, se revelaron como conocedores del tema estudiado (Arenas 2003). De este modo, se trabajó en torno a la indagación de interrogantes concretos, como por ejemplo modalidades de cultivo, tecnología utilizada y productividad de las distintas especies vegetales que se identificaron en contexto arqueológico.

A partir de estas tareas, se pudo observar que actualmente en el Valle de Ambato se emplean tres maneras diferentes de trabajar la tierra. En la primera de ellas, se realiza una rotación de los cultivos, es decir a la siembra del maíz le sigue el próximo año la del poroto y así sucesivamente. En la segunda, se siembra una parcela de maíz o poroto durante cuatro años consecutivos, para luego dejarla descansar por un lapso de dos años. En la restante, se efectúa un cultivo combinado que consiste en sembrar una franja de poroto a ambos extremos de la chacra, lo cual constituye aproximadamente el $10 \%$ del total de la superficie labrada, en el resto del terreno se siembra exclusivamente maíz. Como puede advertirse, la primera y última modalidad comparten la particularidad de que todas las superficies disponibles para el cultivo se trabajan simultáneamente.

En cuanto al riego de los campos, los entrevistados coincidieron en mencionar que para un crecimiento adecuado de las plantas lo óptimo sería realizar tareas de riego con cierta frecuencia, por ejemplo cada 12 o 21 días aproximadamente, sin embargo, se pudo observar que esta situación no siempre se materializa. Dicho de otra manera, la única infraestructura con la que se cuenta para regar las parcelas se restringe a pequeños canales que captan el agua de los arroyos tributarios del río de Los Puestos, los cuales tienen un régimen estacional. En consecuencia, cuando se produce un déficit pluvial de consideración se corre el riesgo de perder la cosecha o incluso de no poder sembrar.

Asimismo, se pudo comprobar que entre los cultivos más difundidos actualmente se encuentra el maíz criollo o 
coyita (Zea mays div. var.). De acuerdo a los agricultores, esta especie goza de una gran aceptación, ya que posee la particularidad de ser sumamente resistente a las heladas y, sobretodo, porque su semilla, generalmente, es "heredada de padres a hijos", como dicen los lugareños.

En todos los campos recorridos, se logró advertir que los entrevistados no emplean ningún tipo de pesticidas ni de fertilizantes modernos, siendo el rastrojo el único método usado para mejorar la tierra.

Otro de los temas explorados consistió en estimar el rendimiento promedio de ciertos recursos cultivables. Sobre este punto, se logró establecer que la productividad del maíz criollo o coyita sería de $1.350 \mathrm{~kg} / \mathrm{ha}$. En tanto, el rendimiento del poroto, la otra especie considerada para este estudio, ascendería a $1.560 \mathrm{~kg} / \mathrm{ha}$. Resulta necesario acotar que en este trabajo no se contemplaron algunas variables que podrían haber incidido en el éxito de la cosecha (i.e. utilización de abono, riego intensivo, plagas de insectos, sequías y heladas), las cuales actualmente se encuentran en proceso de evaluación. Sin embargo, esta situación no invalida de modo alguno los números aquí vertidos.

Finalmente, y una vez estipulado el rendimiento promedio de los recursos cultivables, se indagó sobre el valor energético por kilogramo de cada uno de ellos. En función de la bibliografía existente, se observó que el maíz pisingallo, muy similar al maíz criollo o coyita en lo que respecta a tamaño y peso, posee $3.400 \mathrm{kcal}$ (Muscio 2004). En tanto, que para el poroto el valor energético establecido es de $1.700 \mathrm{kcal}$ (Laguens 1999).

\section{Estimación de la capacidad sustentadora de los sistemas agrícolas}

En base a la información expuesta hasta aquí, se llevaron a cabo una serie de cálculos matemáticos destinados a estimar la capacidad de carga de los sistemas agrícolas ubicados en la Sierra del Ambato.

\begin{tabular}{|c|c|c|c|}
\hline Informante & Localidad & Cultivo & $\begin{array}{l}\text { Rendimiento } \\
\text { por hectárea }\end{array}$ \\
\hline Apolinar Saavedra & Los Varela & Maíz & $750 \mathrm{~kg}$ \\
\hline \multirow[t]{2}{*}{ Alberto Seco } & Los Talas & Maíz & $1.350 \mathrm{~kg}$ \\
\hline & & Poroto & $2.000 \mathrm{~kg}$ \\
\hline Roberto Varela & Los Varela & Maíz & $1.100 \mathrm{~kg}$ \\
\hline Telmo Seco & Los Castillos & Maíz & $800 \mathrm{~kg}$ \\
\hline \multirow[t]{2}{*}{ Jorge Vega } & El Bolsón & Maíz & $1.000 \mathrm{~kg}$ \\
\hline & & Poroto & $1.500 \mathrm{~kg}$ \\
\hline Aída Gordillo & Los Varela & Maíz & $1.350 \mathrm{~kg}$ \\
\hline \multirow[t]{2}{*}{ Adán Aguilar } & El Bolsón & Maíz & $1.650 \mathrm{~kg}$ \\
\hline & & Poroto & $800 \mathrm{~kg}$ \\
\hline Alicia Aguilar & El Bolsón & Maíz & $1.600 \mathrm{~kg}$ \\
\hline Pedro Delgado & Los Varela & Maíz & $1.500 \mathrm{~kg}$ \\
\hline \multirow[t]{2}{*}{ Cristian Vega } & El Bolsón & Maíz & $2.200 \mathrm{~kg}$ \\
\hline & & Poroto & $2.000 \mathrm{~kg}$ \\
\hline \multirow[t]{2}{*}{ Oscar Saavedra } & Los Varela & Maíz & $2.100 \mathrm{~kg}$ \\
\hline & & Poroto & $1.500 \mathrm{~kg}$ \\
\hline Pedro Barrios & Los Talas & Maíz & $800 \mathrm{~kg}$ \\
\hline
\end{tabular}

Tabla 5: Valores promedios de producción de maíz y poroto en el Valle de Ambato.
Para ello, se partió de la premisa que una persona adulta requiere para una correcta alimentación una ingesta diaria de 1.600 kilocalorías (Treacy 1994), es decir, 584.000 kilocalorías anuales. Por lo tanto, si un individuo consumiera únicamente maíz, necesitaría de 171,76 kg de este producto al año. Mientras que si su dieta se centrara exclusivamente en poroto, precisaría una ración anual de $343,53 \mathrm{~kg}$.

A esta altura, resulta significativo señalar que la superficie disponible para el cultivo en la etapa Aguada habría rondado las 494 ha (Figueroa 2008b). Esta cifra será empleada como eje central en la formulación de los cálculos que se desarrollarán en los párrafos subsiguientes.

De este modo, y siguiendo los lineamientos metodológicos propuestos por Treacy (1994), es posible plantear que si en las superficies de producción agrícola de la ladera del Ambato, se hubiese practicado la rotación de cultivo donde, de acuerdo lo expuesto anteriormente, todas las tierras habrían sido cultivadas al mismo tiempo, la producción anual de maíz habría rondado los 666.900 kg (494 ha x 1.350 kg/ha), con lo cual se podría haber abastecido a un total de 3.883 individuos durante un año $(666.900 \mathrm{~kg} / 171,76 \mathrm{~kg})$. Mientras que, si en totalidad de las terrazas de cultivo se hubiese sembrado únicamente poroto, la producción anual habría alcanzado una cifra cercana a los 770.640 kg (494 ha x 1.560 kg/ha), siendo suficiente para mantener a 2.243 individuos durante 365 días $(770.640 \mathrm{~kg} / 343,53 \mathrm{~kg})$.

En cambio, si se hubiese practicado la segunda modalidad de cultivo, es decir, sembrar durante cuatro años maíz o poroto, para posteriormente dejar los terrenos en barbecho, probablemente los números recientemente expuestos sufrirían modificaciones substanciales. Con el propósito de controlar las diferencias de tiempo de barbecho, se usó la siguiente fórmula (Dickson 1980):

$$
T=P\left(\frac{L}{P+F}\right)
$$

Donde:

$\mathrm{T}=$ total de tierra en cultivo durante cualquier año dado $\mathrm{P}=$ número de años de cultivo entre periodos de barbecho $\mathrm{L}=$ total de tierra disponible para el cultivo en unidades cuadradas

$\mathrm{F}=$ número de años de barbecho entre periodos de cultivo

Para los sistemas agrícolas de Ambato esta ecuación se resuelve de la siguiente manera:

$$
\begin{gathered}
T=P \times L /(P+F) \\
4 \times 494 /(4+2)=329 \text { ha }
\end{gathered}
$$

Entonces, a partir de estos cálculos se pudo determinar que, con esta segunda modalidad de cultivo la producción 


\begin{tabular}{lcc}
\hline & Maíz & Poroto \\
\hline Modalidad de cultivo 1 & 3.883 personas & 2.243 personas \\
Modalidad de cultivo 2 & 2.586 personas & 1.494 personas \\
Modalidad de cultivo 3 & \multicolumn{2}{c}{3.721 personas } \\
\hline
\end{tabular}

Tabla 6: Número estimativo de personas mantenidas anualmente por los sistemas agrícolas, según la modalidad de trabajo de la tierra y la clase de cultivo empleado.

de maíz podría haber sido de 444.150 kg, lo cual habría alcanzado para mantener anualmente a 2.586 individuos (329 ha x $1.350 \mathrm{~kg} / \mathrm{ha}=444.150 \mathrm{~kg} / 171,76 \mathrm{~kg}$ ). Por el contrario, si en estas superficies se hubiese sembrado poroto, el rendimiento habría rondado los 513.240 $\mathrm{kg}$. Con este volumen de producción se habría podido alimentar a 1.494 personas durante un año (329 ha $x$ $1.560 \mathrm{~kg} / \mathrm{ha}=513.240 \mathrm{~kg} / 343,53 \mathrm{~kg}$ ).

Finalmente, si se atiende a la ultima modalidad de cultivo registrada para el valle, la cual consiste en sembrar en una parcela $90 \%$ de maíz y el restante $10 \%$ de poroto, se puede señalar que los niveles de producción del maíz (445 ha $=600.750 \mathrm{~kg}$ ) y del poroto (49 ha $=76.440$ ) podrían haber sido suficientes como para sustentar a 3.498 y 223 individuos, respectivamente. Es decir, el rendimiento combinado de ambos cultivos en las 494 ha consideradas en este trabajo, habrían posibilitado la alimentación de 3721 personas durante 12 meses.

\section{Consideraciones finales}

Tal como se señaló al comienzo de este trabajo, el objetivo perseguido fue estimar la capacidad sustentadora de los sistemas agrícolas de la ladera del Ambato, para lo cual resultan de suma trascendencia las aproximaciones demográficas efectuadas para el sector central del valle. Sobre este aspecto, Gordillo (2003) plantea que existen al menos tres métodos diferentes para llevar a cabo cálculos demográficos:

1) Relacionando el área total de vivienda con el espacio requerido por persona (Casselberry 1974; Narroll 1962).

2) Multiplicando la cantidad de viviendas o estructuras asociadas directamente a cada unidad domestica, por el promedio de sus habitantes (Hill 1970; Milisauskas 1986; Nelson 1999).

3)Vinculando el tamaño del núcleo familiar con las superficies de las viviendas (Hassan 1978; Renfrew y Bahan 1993).

Los cálculos demográficos sobre el tamaño de la población para el Valle de Ambato, se llevaron a cabo considerando el segundo de estos métodos. Por esta razón, resultó indispensable establecer no solo el número de unidades residenciales presentes en el área de estudio, sino también estimar el promedio de habitantes por cada una de ellas.

Con respecto al tamaño del núcleo familiar se puede decir que una gran parte de la bibliografía especializada, procedente de diferentes partes del mundo, coincide en que una unidad domestica generalmente alberga en promedio a seis individuos (Hill 1970; Milisauskas 1986; Raffino 1973; entre otros). Mientras que, para determinar el número de viviendas presentes en el área, se recurrió a otros trabajos del equipo del Proyecto Arqueológico Ambato, pudiéndose así conocer que de 139 sitios registrados, sólo para el sector central del valle, 88 corresponden a unidades habitacionales (Assandri 1999, 2006; Assandri y Laguens 2003). A partir de estos números, se puede plantear que para el caso en estudio la población estimada rondaría las 528 personas (88 unidades habitacionales $X$ 6 individuos).

Entonces, si se atiende a los volúmenes de producción agrícola estimados en este trabajo, más allá de que varíen en función a la modalidad de cultivo que se implemente y al recurso vegetal involucrado (Tabla 6), se puede concluir que los mismos habrían sido suficientes como para alimentar a la totalidad la población de este sector del valle. Esta hipótesis, se refuerza si se tiene en cuenta que la dieta de una persona se encuentra integrada por otros alimentos que no sólo derivan de la producción agrícola. En Ambato, las excavaciones llevadas a cabo en unidades domésticas y ceremoniales como por ejemplo Piedras Blancas y La Rinconada posibilitaron identificar la presencia de abundantes restos de semillas de chañar, algarrobo y mistol (Gordillo 2005; Gordillo y Ares 2005; Pochettino 2000). En tanto, los restos faunísticos identificados, con evidencias de haber sido consumidos, comprenden camélidos, cérvidos y dasipódidos, entre otros (Bonnín 2000; Dantas 2007). Además, aún falta procesar los datos derivados de las prospecciones efectuadas sobre la sierra de la Graciana, donde los recorridos permitieron identificar la presencia de infraestructura hidroagrícola de similares características a las descriptas en este trabajo (Cruz 2008; Figueroa 2008; Herrero y Ávila 1993), con lo cual los rindes se incrementarían notablemente.

En definitiva, este panorama refleja que la producción agrícola en Ambato, habría alcanzado valores lo suficientemente importantes para ser utilizados no solo como alimentos, sino también como productos de intercambio o quizás como acumulación de excedentes, por parte de un determinado sector de la población. Esta ultima situación, en una sociedad internamente diferenciada, como es el caso de Aguada de Ambato, podría haberse transformado en una de las causas que posibilitaron el establecimiento y/o mantenimiento de relaciones desigualitarias.

Córdoba, 18 de octubre de 2009

\section{Agradecimientos}

Al Dr. Andrés Laguens por la lectura y sugerencias 
realizadas a este trabajo. A la Lic. Mariana Dantas, Lic. Eduardo Pautassi, Prof. José Siles y Dr. Walter Sinay, quienes participaron en las discusiones sobre este tema. Al Lic. Henrik Lindskoug, Rita Bargas y Soledad Salega que colaboraron en las campañas de prospección. A la Ingeniera Agrónoma Yamila Sobh de la Estancia La Rinconada y al Ingeniero Agrónomo Mario Alloatti, por el aporte desinteresado de sus conocimientos. A todos los agricultores de Los Varela, Los Castillos, Los Talas y El Bolsón.

\section{Bibliografía}

Abbot, M. B., M. W. Binford, M. Brenner y K. R. Kelts. 1997. A 3500 14C yr High-Resolution Record of WaterLevel Changes in Lake Titicaca, Bolivia/Peru. Quaternary Research, 47: 169-180.

Albeck, M. E. 1993. Contribución al estudio de los sistemas agrícolas prehispánicos de Casabindo (Puna de Jujuy). Tesis Doctoral, Facultad de Ciencias Naturales y Museo, Universidad Nacional de la Plata, Argentina, 165 páginas.

Albeck, M. E. 2003/2005. Sistemas agrícolas prehispánicos: la búsqueda de indicadores cronológicos y culturales. Cuadernos del Instituto Nacional de Antropología y Pensamiento Latinoamericano, 20: 13-26.

Ardissone, R. 1944. Andenes en la Cuenca del Torrente de las Trancas (Provincia de Catamarca). Relaciones de la Sociedad Argentina de Antropología, IV: 93-109.

Arenas, P. 2003. Etnografía y alimentación entre los Toba-Ñachilamole'ek y Wichí-Lhuku'tas del Chaco central (Argentina), Pastor Arenas, Buenos Aires.

Assandri, S. 1999. Procesos de Complejización Social y Organización Espacial en el Valle de Ambato, Catamarca, Argentina. Tesis de Maestría, Universidad Internacional de Andalucía, España, 129 páginas.

Assandri, S. 2006. Técnicas de Análisis Espacial de la Cultura La Aguada en el Valle de Ambato, Catamarca, Argentina. Actas de la IV Mesa Redonda: La Cutura de La Aguada y su Dispersión, pp. 107-126, San Pedro de Atacama.

Assandri, S. y A. Laguens. 2003. Asentamientos aldeanos Aguada en el Valle de Ambato. Actas del XIII Congreso Nacional de Arqueología Argentina, Vol. 3: 31-40, Córdoba.

Baied, C. 1999. Distribución actual de matorrales altoandinos de Polylepis en los Andes centro-sur: Oscilaciones climáticas y el impacto de la actividad humana en el pasado. En C. Aschero, M. A. Korstanje y P. Vuoto (ed.), Los tres reinos: prácticas de recolección en el cono sur de América, pp. 129-139. Instituto de Arqueología y Museo, Universidad Nacional de Tucumán, Tucumán.
Barlow, K. R. 2002. Predicting maize agriculture among the Fremont: an economic comparison of farming and foraging in the American Southwest. American Antiquity, 67(1): 65-88.

Binford, L. 1978. Nunamiut ethnoarchaeology, Academic Press, New York.

Binford, L. 1983. Working at archaeology, Academic Press, New York.

Binford, M., A. L. Kolata, M. Brenner, J. W. Janusek, M. T. Seddon, M. Abbott y J. H. Curtis. 1997. Climate Variation and the Rise and Fall of an Andean Civilization. Quaternary Research, 47: 235-248.

Bonnin, M. 2000. Patrones de consumo de animales en el Valle de Ambato, Catamarca. Trabajo presentado en la IV mesa Redonda La Cultura de la Aguada y su dispersión, San Pedro de Atacama.

Borrero, L. y H. Yacobaccio. 1989. Etnoarqueología de Asentamientos Aché. Journal De La Societé Des Américanistes, LXXV: 7-33.

Cassellberry, S. 1974. Further Refinements of Formulae for Deternining Site Population from Floor Area. World Archaeology, 6: 118-122.

Cruz, P. 2002. Entre pumas y jaguares. Algunas reflexiones acerca de la iconografía del valle de Ambato (CatamarcaArgentina). Revista Andina, 34: 217-236.

Cruz, P. 2004. Sous le signe du jaguar. Archeologie du bassin de Los Puestos (Dpto. Ambato-Catamarca). Tesis Doctoral, Universidad de Paris I Pantheon Sorbonne, Francia, 559 páginas.

Cruz L., A. y J. Palerm V. 2001. Abasto y excedentes en la producción agrícola a través de la historia. En De La Isla de Bauer, Tijerina, Rodríguez, Muñoz, Escobedo (ed.), Memorias IV Simposio Internacional y V Reunión Nacional sobre Agricultura Sostenible 1999, pp. 453-460.

Dantas, M. 2007. El registro faunístico como indicador de diferenciación social. El caso de Piedras Blancas, Valle de Ambato, Catamarca (siglos VI- XI d.C.). Trabajo presentado en el XVI Congreso Nacional de Arqueología Argentina, San Salvador de Jujuy.

Dantas, M. y G. G. Figueroa. 2009. Terrazas y corrales como espacios integrados de producción agro-pastoril en el Valle de Ambato, Catamarca, Argentina (s. VI-XI d.C.). Relaciones de la Sociedad Argentina de Antropología, en prensa.

de la Orden, E. y A. Quiroga. 1997. Fisiografía y vegetación de la cuenca del río Los Puestos. Departamento 
Ambato. Catamarca. Revista de Ciencia y Técnica, IV (4): 27-45.

Denevan, W. 1980. Tipología de las configuraciones agrícolas prehispánicas. América indígena, XL (4):619-652.

Dickson, B. 1980. Ancient agriculture and population at Tikal, Guatemala: an application of linear programming to the simulation of an archaeological problem. American Antiquity, 45 (4): 697-712.

Donkin, R. 1979. Agricultural Terrasing in the Aboriginal New World, University of Arizona Press, Tucson.

Erickson, C. 1980. Sistemas agrícolas prehispánicos en los llanos de Mojos. América Indígena, XL (4): 731-755.

Erickson, C. L. 1992. Prehistoric Landscape Management in the Andean Highlands: Raised Field Agriculture and its Environmental Impact. Population and Environment: A Journal of Interdisciplinary Studies, 13 (4): 285-300.

Fabra, M. 2002. Producción tecnológica y cambio social en sociedades agrícolas prehispánicas (Valle de Ambato, Catamarca). Tesis de Licenciatura, Escuela de Historia, Facultad de Filosofía y Humanidades, Universidad Nacional de Córdoba, Argentina, 304 páginas.

Fabra, M. 2006. Estimaciones sobre la inversión artesanal en la producción cerámica: sitio arqueológico Piedras Blancas (Valle de Ambato, Catamarca, Argentina). Actas de la IV Mesa Redonda: La Cutura de La Aguada y su Dispersión, pp. 7-18, San Pedro de Atacama.

Figueroa, G. G. 2008a. Los sistemas agrícolas del Valle de Ambato, Catamarca, siglos VI a XI d.C. Intersecciones en Antropología, 9: 365-367.

Figueroa, G. 2008b. Informe de avance de las actividades desarrolladas durante el segundo año de beca de doctorado de SECyT. Copia disponible en Museo de Antropología, Universidad Nacional de Córdoba, Córdoba.

Gordillo, I. 2003. Organización socioespacial y religión en Ambato, Catamarca. El sitio ceremonial de La Rinconada. Tesis Doctoral, Facultad de Filosofía y Letras, Universidad de Buenos Aires, Argentina, 493páginas.

Gordillo, I. 2005. Dimensión temporal del sitio La Rinconada. Su interpretación y aportes a la historia del Periodo Medio. Actas de la V Mesa Redonda de la cultura de La Aguada y su dispersión, pp. 159-171, La Rioja.

Gordillo, I. y L. Ares. 2005. Ingresando a los patios de La Rinconada (Ambato, Catamarca). Actas de la V Mesa Redonda de La cultura de La Aguada y su dispersión, pp. 211-225, La Rioja.
Hassan, F. 1978. Demographic Archaeology. En M. B. Schiffer (ed.), Advances in Archeological Method and Theory, Vol.1, pp.49-103, Academic Press, New York.

Hernando, A. 1995. La Etnoarqueología hoy: una vía eficaz de aproximación al pasado. Trabajos de Prehistoria, 52 (2): 15-30.

Herrero, R. y A. Ávila. 1993. Aproximación al estudio de los patrones de asentamiento en el Valle de Ambato (Prov. de Catamarca, Argentina). Publicaciones 6, Serie Investigaciones $\mathrm{N}^{\circ} 4$, Instituto de Arqueología, U. N. de Tucumán.

Hierling, J. 2000. Prospección y relevamiento de construcciones agrícolas del valle de Ambato. Informe a SECyT. Copia disponible en Museo de Antropología, Universidad Nacional de Córdoba, Córdoba.

Hill, J. 1970. Broken K Pueblo: Prehistoric Social Organization in the American Southwest. Anthropological Papers of the University of Arizona 18, University of Arizona Press.

Hodder, I. 1982. Symbols in Action: Ethnoarchaeological Studies of Material Culture, Cambridge.

Kriscautzky, N. 1995. Arqueología del Formativo Inferior del Valle de Catamarca. Revista de la Secretaría de Ciencia y Técnica UNCa, 2: 65-82.

Kriscautzky, N. 2000a. Nuevos aportes en la arqueología del Valle de Catamarca. Shincal 6: 27-34.

Kriscautzky, N. 2000b. Sistemas productivos y estructura arqueológicas relacionadas con la producción agropecuaria en el Valle de Catamarca. Shincal 6: 65-70.

Kuznar, L. 2001. Ethnoarchaoelogy of Andean South America. International Monographs in Prehistory, Ethnoarchaeological Series 4, Michigan.

Laguens, A. 1999. Arqueología del contacto hispanoindígena. Un estudio de cambios y continuidades en las Sierras Centrales de Argentina. BAR, International Series 801, Oxford.

Laguens, A. 2004. Arqueología de la diferenciación social en el valle de Ambato, Catamarca, Argentina (s. II-VI d.C.): El actualismo como metodología de análisis. Relaciones de la Sociedad Argentina de Antropología, XXIX: 137-161.

Laguens, A. 2006. Continuidad y ruptura en procesos de diferenciación social en comunidades aldeanas del Valle de Ambato, Catamarca, Argentina (s. IV-X d.C.). Chungara, 38 (2): 211-222. 
Marconetto, M. B. 2006. Casas de Fuego. Análisis antracológico de los restos de techos carbonizados del sitio Piedras Blancas (Depto. Ambato, Provincia de Catamarca, Argentina). Actas de la IV Mesa Redonda: La Cutura de La Aguada y su Dispersión, pp. 19-28, San Pedro de Atacama.

Marconetto, M. B. 2008. Recursos forestales y el proceso de diferenciación social en tiempos prehispánicos en el valle de Ambato, Catamarca, Argentina. British Archaeological Reports- South American Archaeology Series, Oxford.

Markgraf, V. 1985. Paleoenvironmental history of the last 10000 years in Northwestern Argentina. Zentralhlatt fur Geologie und Palantologie, 11-12: 1739-1749.

Milisauskas, S. 1986. An Analysis of Linear culture longhouses at Olszanica B1, Poland. World Archaeology, 4: 57-74.

Morlans, M. y B. Guichón. 1995. Reconocimiento ecológico de la Provincia de Catamarca I: Valle de Catamarca. Vegetación y Fisiografía. Revista de Ciencia y Técnica, $1(1)$.

Moseley, M. E., D. J. Nash, P. R. Williams, S. D. deFrance, A. Miranda, y M. Ruales. 2005. Burning down the brewery: Establishing and evacuating an ancient imperial colony at Cerro Baúl, Peru. Proc. Natl. Acad. Sci. USA, 102 (48): 17264-17271.

Muscio, H. 2004. Dinámica Poblacional y Evolución durante el Período Agroalfarero Temprano en el valle de San Antonio de los Cobres, Puna de Salta, Argentina. Tesis doctoral, Facultad de Filosofía y Letras, Universidad de Buenos Aires, Argentina, 523 páginas.

Narrol, R. 1962. Floor Area and Settlement Population. American Antiquity, 27: 587-589.

Nelson, M. 1999. Mimbres During the Twelfth Century. Abandonment continuity and Reorganization. The University of Arizona Press. Tucson.

Nielsen, A. 1997. El tráfico caravanero visto desde La Jara. Estudios Atacameños, 14: 339-371.

O'Connell, J. 1995. Ethnoarchaeology Needs a General Theory of Behavior. Journal of Archaelogical Research, 3(3): 205-255.

Pérez Gollán, J. 1991. La Cultura de la Aguada vista desde el Valle de Ambato. Publicaciones del CIFFyH Arqueología, 46: 157-174.

Pérez Gollán, J. 2000. El jaguar en llamas (La religión en el antiguo Noroeste Argentino). En Myriam N. Tarragó (ed.), Nueva Historia Argentina. Tomo 1: Los pueblos originarios y la Conquista, pp. 229-256, Sudamericana, Buenos Aires.

Pochettino, M. 2000. Informe técnico de determinación de vegetales. Copia disponible en Museo de Antropología, Universidad Nacional de Córdoba, Córdoba.

Politis, G. 2002. Acerca de la Etnoarqueología de América del Sur. Horizontes Antropológico, 8(18): 61-91.

Politis, G. 2004. Tendencias de la etnoarqueología en América del Sur. En Gustavo Politis y Roberto Peretti (ed.), Teoría arqueológica en América del Sur, Numero 3, pp. 85-119, INCUAPA, Olavarría.

Puentes, H. 2003. Los primeros tiempos del formativo en el valle de Catamarca, control de cuenca, manejo hidráulico y uso del espacio, un caso de estudio: sitio El Tala (Departamento Capital, Catamarca), CENEDIT, Catamarca.

Puentes, H. 2005. Economía agrícola prehispánica. Distribución y función de las estructuras destinadas a la producción en el espacio serrano durante período Formativo. Actas de la V Mesa Redonda de La cultura de La Aguada y su dispersión, pp. 199-210, La Rioja.

Raffino, R.1973. Agricultura hidráulica y simbiosis económica demográfica en la Quebrada del Toro, Salta, Argentina. Revista del Museo de La Plata (Nueva Serie), Sección Antropología, VII: 297-332.

Ratto, N. 2003. Estrategias de caza y propiedades del registro arqueológico en la Puna de Chaschuil (Dpto. Tinogasta, Catamarca). Tesis Doctoral, Facultad de Filosofía y Letras, Universidad de Buenos Aires, Argentina.

Renfrew, C. y P. Bahn. 1993. Archaeology: theories, methods, and practices, Themesand Hudson, London.

Saravia Toledo, C. 1995. Recuperación y conservación de áreas críticas en la subcuenca del río Los Puestos. Primer Informe Parcial. Disponible en Secretaria de Medioambiente de la Provincia de Catamarca, Catamarca.

Thompson, L., M. Davis y E. Moseley-Thompson. 1994. Glacial records of global climate: A 1500 year tropical ice core record of climate. Human Ecology, 22(1): 83-95.

Treacy, J. 1994. Las chacras de Coporaque. Andenería y riego en el Valle del Colca, Instituto de Estudios Peruanos, Lima.

Valero Garcés, B., A. Delgado Huertas, N. Ratto, A. Navas y L. Edwards. 2000. Paleohydrology of Andean Saline Lakes from Sedimentological and Isotopic Records, Northwestern Argentina. Journal of Paleolimnology, 243: 343-359. 
Wylie, A. 1982. An analogy by any other name is just as analogical: a commentary on the Gould-Watson dialogue. Journal of Anthropological Archaeology, 1: 382-401.

Wylie, A. 1985. The reaction against analogy. Advances in Archaeological Method and Theory 8: 63-111.

Yacobaccio, H., C. Madero, M. Malmierca. 1998. Etnoarqueología de Pastores Surandinos, Grupo Zooarqueología de Camélidos, Buenos Aires. 